\title{
CYBER PHYSICAL SYSTEMS: A NEW FRONTIER OF ARTIFICIAL INTELLIGENCE: SUMMARY PAPER
}

\author{
Dr. Sabah Mohammed ${ }^{1}$ and Dr. Jinan Fiaidhi ${ }^{2}$ \\ ${ }^{1}$ Department of Computer Science \\ Lakehead University, Ontario Canada \\ ${ }^{2}$ Department of Computer Science \\ Lakehead University, Ontario Canada \\ ${ }^{1}$ mohammed@lakeheadu.ca, ${ }^{2}$ jfiaidhi@lakeheadu.ca
}

\begin{abstract}
In the last two decades we been witnessing the advancement of many applications based on the notion of embedded systems. However embedded systems have contributed only to a small-scale and standalone applications. With these systems electronic engineers design a chip board for accommodating such embedded systems following the principle of throwing enough mud at the wall and some of it will stick. Actually the recent advances in computing and communication technologies provided the wide adoption of internet-connected devices are pulling the concept of Cyber-Physical Systems (CPS) as the pervasive bridge between the real world (of people, equipment's and embedded systems) and information (the cyber world) technologies.
\end{abstract}

Keywords - Cyber-Physical Systems, Artificial Intelligence, Internet of Things, Web of Things

\section{INTRODUCTION}

Internet of Things, Web of Things and "Smart Systems" environments are some examples of these systems, which have become exciting application fields for artificial intelligence. AI methodologies, models and tools have to face numerous challenges when facing the new requirements of CPS. For example, designers have to pay particular attention to non-functional requirements as the limited resources (memory, CPU), energy consumption, safety of equipment and people, delay-tolerant communication, autonomy in decision making, etc. Many scholars tag CPS as the fourth industrial revolution as illustrated in Figure 1. 




Fig. 1. The Timeline of Industrial Revolutions.

Cyberphysical systems are engineered systems that are built from and depend upon, the seamless integration of computational algorithms and physical components like robots and other machinery. Generally, there are multiple decision-making entities (multi-agents) in such systems. In manufacturing CPS are called Smart Factories [1]. AI is rapidly becoming an essential technology that's helping manufacturers inject next-level automation into their processes. In factories, deep learning can be leveraged in several areas, including planning, scheduling, and predictive maintenance [2]. AI has the potential to radically improve the efficiency and effectiveness of decision-making in highly complex data environments all while embedding an ever-increasing amount of knowledge within these intelligent systems. Figure 2 illustrate the new wing of AI which include the focus on CPS [3].

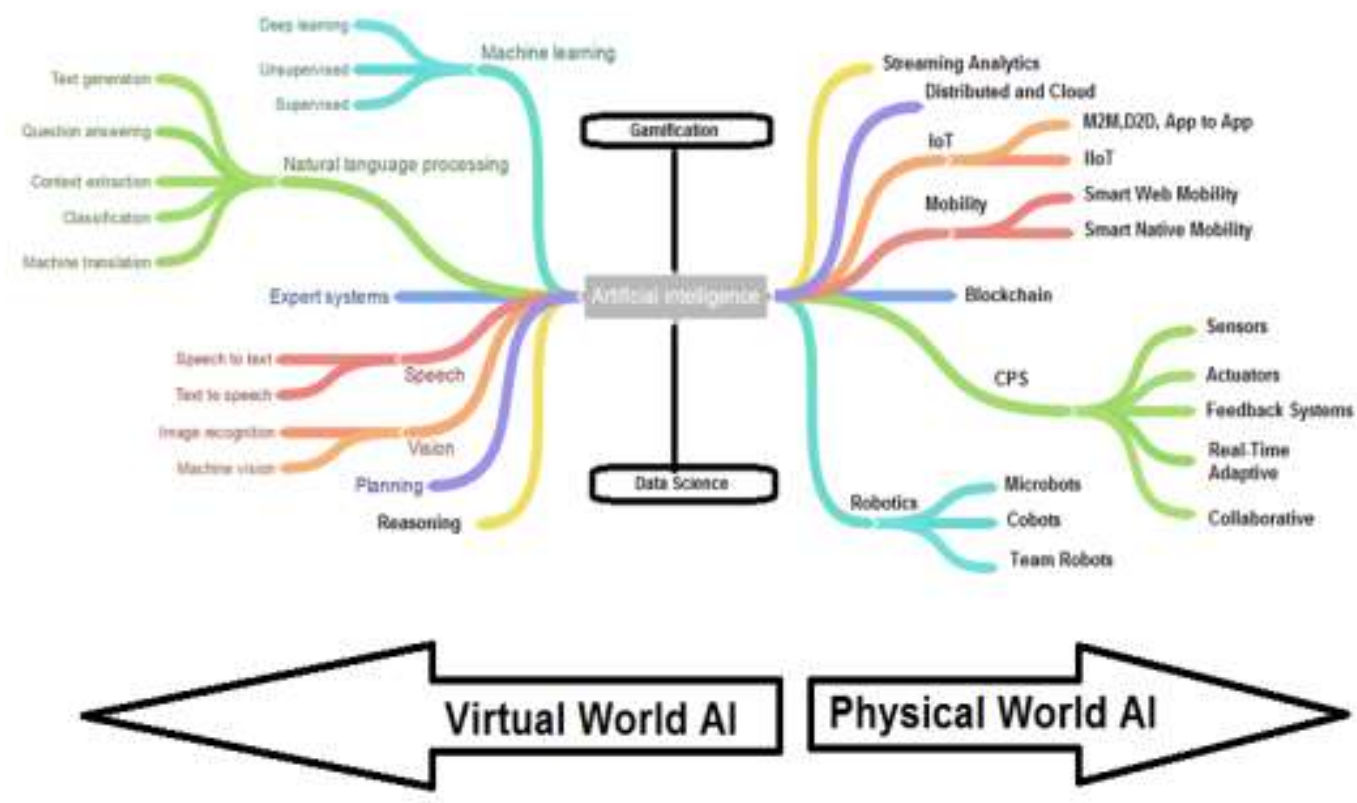

Fig. 2. AI Role in Bridging the Virtual and Physical Words. 
AI has already begun playing an important role in automation utilizing robotics. In agriculture, for instance, John Deere now produces unmanned farming vehicles with highly autonomous capabilities. Applying machine learning and cloud services to robotics automation creates new opportunities for manufacturers to achieve higher reliability, increase productivity and more. In fact, artificial intelligence has brought us into a new dimension that extends beyond the walls of one plant. While robots have occupied the factory floor for some time, today's robots no longer perform monotonous, mechanical tasks. They are smart participants in the CPS revolution at its core, the linking of the reallife smart factory with the power of virtual reality.

\section{THE ROLE OF INTELLIGENT ROBOTS?}

Robots are coming the new workforce of manufacturing to work alongside humans. Traditionally robots are classified in manufacturing as industrial robots and typically they are sort of articulated arms featuring six axes of motion (6 degrees of freedom). This traditional design allows maximum flexibility. There are six main types of industrial robots: Cartesian, SCARA, Cylindrical, Delta, Polar and Vertically Articulated. However, there are several additional types of robot configurations. Each of these types offers a different joint configuration. The joints in the arm are referred to as axes. Typical applications of industrial robots include welding, painting, assembly, pick and place for printed circuit boards, packaging and labeling, palletizing, product inspection, and testing; all accomplished with high endurance, speed, and precision.

Robot adoption in the manufacturing industry increased by an annual global average of 12\% between 2011 and 2016, concentrated heavily in the automotive and electronic / electrical manufacturing sectors. According to the International Federation of Robotics, more than 3 million industrial robots will be in use in factories around the world by 2020 [4]. Global sales of industrial robots reached the new record of 387,000 units in 2017. That is an increase of 31 percent compared to the previous year (2016: 294,300 units). China saw the largest growth in demand for industrial robots, up 58 percent. Sales in the USA increased by 6 percent - in Germany by 8 percent compared to the previous year. These are the initial findings of the World Robotics Report 2018 [5]. However, the customer demand for greater product variety is driving an increase in low-volume, high-mix manufacturing. Industrial robots although they can be programmed to work for different settings, they cannot adapt and rapidly repurpose their production facilities in the same demanding speed. The need for smart factories, in which machines are digitally linked, is ever growing with the new notion of CPS revolutions [1]. Smart factories create a supply chain that shorten product development time, repurposed quickly, reduce product defects and cut machine downtime. The smart factory ecosystem is used to describe the seamless communication between all levels of extreme automation to a fully connected and flexible system — one that can use a constant stream of data from connected operations and production systems to learn and adapt to new demands. Central to the issue of such ecosystem is the notion of "Smart Manufacturing Units (SMU)" which signifies the opportunity to drive greater value both within the four walls of the factory and across the supply network. Every SMU is a flexible system that can self-optimize performance across a broader network, self-adapt to and learn from new conditions in real or near-real time, and autonomously run entire production processes. SMUs can operate within the four walls of the factory, but they can also connect to a global network of similar production systems and even to the digital supply network more broadly. SMU enables all information about the manufacturing process to be available when it is needed, where it is needed, and in the form that it is needed across entire manufacturing supply chains, complete product lifecycles, multiple industries, and small, medium and large enterprises. In fact the new advances in collaborative robots and assistive technologies such as exoskeletons expand the scope of tasks robots can perform in support of the SMUs integration and adaptivity. Other 
significant advances are also adding more positive effects in era of smart factories like 'Cloud robotics', 'Robot as Service' and Microbots. In the next section we shed some light on some of these advances that have important effect in increasing the uptake of robots for extreme automation. However, we may address some these issues in our next columns as they relate to other aspects of the extreme automation initiative.

The current industrial robots are still pretty rudimentary-and expensive. They are typically been large, caged devices that perform repetitive, dangerous work in lieu of humans. These robots suffers from many problems like the vision issues as robots do not have the ability to identify and navigate around objects (including people) and dexterity issues as their gripping, maneuvering and mechanical capabilities are still limited. As the digital technology advances and development of autonomous vehicles have driven down costs of off-the-shelf hardware, smaller, more dexterous robots have come onto the factory floor. Actually low-cost robot designs poured in from around the world in response to many initiatives around the world like the Kickstarter campaign (www.kickstarter.com) advocating for a basic low-cost industrial arm for nontraditional markets like Fablabs. These lighter weight, lower cost robots can be outfitted with sensors that allow them to work collaboratively alongside humans in industrial settings, creating "cobots" or "FabLab robots" - robots that can perform tasks like gripping small objects, seeing, and even learning to tackle "edge cases." Niryo One (https://niryo.com/) is one example from the Kickstarter campaign where the low-cost industrial robot is a 6 axis robotic arm, made for makers, education, and small factories. The robot is 3D printed and powered by Arduino, Raspberry Pi, and Robot Operating System. STL files and code on the robot are open source. Niryo One is also the first industrial robot that can be connected to your home and can be accessed and controlled via the Internet. The introductory price of Niryo One is less than $\$ 1,000$. There are other examples like the Franka Emika, (www.franka.de) a rather remarkable cobot arm. It's designed to be easy to set up and program and can operate right next to people, assisting them with tasks without posing a risk and it can build copies of itself. Now all the big industrial robot makers are trying to develop their own cobots, but the most innovative designs have come from startups like the Rethink Robotics who pioneered the Baxter dual-arm robot in 2012, and later the single-arm robot called Sawyer. Both co-bots are simple to use as their Intera software platform provides a train by demonstration experience unlike any other cobot. You can train these cobots by simply moving its arm and demonstrating the movements. With the introduction of Intera 5, Rethink Robotics have created the world's first smart robot that can orchestrate the entire work cell, removing areas of friction and opening up new and affordable automation possibilities for manufacturers around the world. Intera 5 is driving immediate value while helping customers work toward a smart factory, and providing a gateway to successful industrial internet of things (IIoT) for the first time [6].

Collaborative robots are now defined by ISO 10218, which defines five main collaborative robot features: Safety monitored stop, speed and separation monitoring, power and force limiting, hand guiding and risk assessment [7].

\section{Power \& force limiting}

Robot force is limited through electrical or mechanical means;

2. Safety monitored stop

Robot stops when the user enters area and continues when they exit;

3. Speed \& position monitoring

Robot slows down when user nears and stops if the user gets too close;

\section{Hand guiding}

The user is in direct contact with the robot while they are guiding and training it. 


\section{Risk Assessment}

Risk assessment of the application should be done to determine all possible risks and proper devices and procedures to mitigate the risk should be implemented.

Many cobots were successful in following these guidelines and achieving the highest sales among manufacturing companies like the Universal Robots cobots (https://www.universal-robots.com/).

There are many other low-cost industrial cobots that you can find by a simple search like KINOVA Ultra lightweight robotic arm (www.kinovarobotics.com) which is weighing in at just $4.4 \mathrm{~kg}$ and give the workers a performance, flexibility and ease all in on with plugand-play option developed with open architecture and compatible with ROS. However, the main challenge with cobots is not just the hardware and following the ISO 10218 standard but also the software to make it easily accessible to nonexperts and smart enough to collaborate with other human co-workers and cobots. What it does mean is that cobots need to be equipped with sensors, smart technologies and algorithms which are linked with the IoT and/or specific systems like the vision system. Vision systems allowing robots to identify and safely navigate around objects were largely an afterthought. In recent years, vision hardware (such as lidar) has become much cheaper, more effective, and subsequently more widespread. Lidar works much like radar, but instead of sending out radio waves it emits pulses of infrared light - aka lasers invisible to the human eye - and measures how long they take to come back after hitting nearby objects. It does this millions of times a second, then compiles the results into a so-called point cloud, which works like a 3-D map of the world in real time - a map so detailed it can be used not just to spot objects but to identify them. Once it can identify objects, the cobot can predict how it will behave, and thus how it should move. A good example of such cobot is the CES2018 for its ping-pong playing perfection (https://www.cbinsights.com/company/omron). However, the capability of such cobot in distinguishing what an object is and deciding how to move it is still a challenge or bottleneck. What is needed is a cobot that recognizes people's movements and gestures as well as the objects it is surrounded by including other cobots. A cobot that allows real-time mapping of the workspace, being in this way able to adapt the movements of the robots to which it is connected. Smart cobots according to DARPA [9] need to be a small object of moderate weight capable of instantaneously recognizing and interpreting everything that is happening in the workspace, enabling the cobot to become more flexible and to react to sudden changes in the work process. DARPA announced a new program called SHRIMP: SHort-Range Independent Microrobotic Platforms. The goal is "to develop and demonstrate multi-functional micro-to-milli robotic platforms for use in natural and critical disaster scenarios." To enable robots that are both tiny and useful, SHRIMP will support fundamental research in the component parts that are the most difficult to engineer, including actuators, mobility systems, and power storage. To help overcome the challenges of creating such smart microbots the first required stage is to enforce such microbots with optimized size, weight, and power or what is known as SWaP which are just some of the constraints that very small robots operate under. One of the very first microbot was a "push button" that can push nearly any mechanical button and makes such dumb buttons smart through controlling it via your smartphone or a tablet from any distance through WiFi connection. The push button microbot can turn on any device like the coffee maker at a scheduled time or it can be controlled wirelessly.

The next expected stage is to provide such microbots with smart algorithms to enable their work and collaboration towards achieving common goals. For example at the University of California, San Diego, a research lab managed to develop a tiny 3D-printed robotic fish smaller than the width of a human hair that can be used to deliver drugs to specific places in our bodies and sense and remove toxins. These microfishes are selfpropelled, magnetically steered, and powered by hydrogen peroxide nanoparticles [8]. Actually Artificial intelligence (AI) and machine learning capabilities have been quickly making their way into industrial robotics technology and microbots. In the never-ending 
quest to improve productivity, manufacturers are looking to improve on the rigid, inflexible capabilities of legacy industrial robots and make these robots smart, small and effective. Good examples on adding such smart capabilities to the existing cobots or microbots are the FANUC Intelligent Edge Link and Drive (FIELD) industrial internet of things platform to create an intelligent system of industrial collaborating or team robots [9]. Kuka and Huawei [10] signed a deal to develop what could be another global network - built on the industrial internet of things - to enable the connection of robots across many factories. The companies say they plan to integrate artificial intelligence and deep learning into the system. Moreover, if the power of robot team work is combined with a ground-breaking technology like blockchain it will make the industrial robotic operations more secure, flexible, autonomous, and even profitable [11]. Figure 2 illustrates the trends of using industrial robots starting from the traditional industrial robots and ending with team robots. The enabling technologies for such trend include smart algorithms, internet of everything, mechatronics and blockchains.

\section{THE ROLE OF COMPUTER VISION AND IMMERSIVE VIRIAL REALITY?}

Even the most eagle-eyed human inspector would fail at finding flaws half the width of a human hair, for example. But a machine equipped with a camera many times more sensitive than the naked eye won't miss a beat. The trick is to take what would seem like the next logical step-sending those images to a person to make judgments and corrections - and hand that over to the machine as well. Landing.ai, a startup formed by Silicon Valley veteran Andrew $\mathrm{Ng}$, has developed machine-vision tools to find microscopic defects in products such as circuit boards at resolutions well beyond human vision, using a machine-learning algorithm trained on remarkably small volumes of sample images. Once on line, the computer not only "sees," but also processes the information and learns from what it sees. If it spots a problem or defect, it sends an immediate alert, an AI process known as "automated issue identification.[12]" By implementing an immersive virtualreality environment, some AI applications claim it is possible to test products or retail ideas that have not been brought to on the market, putting them on a virtual shelf to study consumer reactions and behavior to real-time merchandising. By integrating an eye-tracker with a head mount display, these companies claim it may also be possible to monitor the consumer gaze and behavior in regard to certain products. According to a study by Deloitte, worldwide revenues from the virtual and augmented reality (VR/AR) market will grow from $\$ 5.2$ billion in 2016 to more than $\$ 162$ billion in 2020. This represents a combined annual growth rate of 181.3 percent from 2015 to 2020. To date, eight of the world's top 10 technology firms have invested in VR, including Apple, Google, Microsoft, Facebook, Samsung, and IBM. In retail specifically, some of the biggest supermarket chains have built their own technology labs to explore how VR and other new technologies can bring benefits to the business. In summary Artificial Intelligence (AI), Robotics, CPS and Artificial Reality (AR) are changing our future.

\section{REFERENCES}

[1] Fiaidhi, J., Extreme Automation: A New Game-Changing Technology. IEEE IT Professional, 20(2), 2018 pp.88-90.

[2] Edwards, J., Building a Smart Factory with AI and Robotics, WHITEPAPER, Robotics Business Review, Feb 2018, Available Online: https://www.roboticsbusinessreview.com/wpcontent/uploads/2018/02/RBR_BuildingAI_WP3.pdf2.

[3] Fiaidhi, J., Mohammed, S. and Mohammed, S., The Robotization of Extreme Automation: The Balance Between Fear and Courage. IEEE IT Professional, 20(6), 2018, pp.87-93.

[4] IFR 2018 Positioning Paper, Robots and the Workplace of the Future, International Federation of Robotics, $\quad$ March 2018, Available 
https://ifr.org/downloads/papers/IFR_Robots_and_the_Workplace_of_the_Future_Positioning_Paper.pd $\mathrm{f}$

[5] Steven Crowe, Industrial robot sales increase 29\% worldwide, IFR reports, June 20, 2018, Available Online: https://www.therobotreport.com/industrial-robot-sales-29-worldwide/

[6] Kelly McSweeney, Rethink Robotics rethinks its software, ZDNet Blog, February 8, 2017, Available Online: https://www.zdnet.com/article/rethink-robotics-rethinks-its-software/\#ftag=CAD-00-10aag7e

[7] The Economist SPECIAL REPORT, Collaborative manufacturing: All together now, Apr 21st 2012, Available Online: https://www.economist.com/special-report/2012/04/21/all-together-now

[8] IEEE Spectrum, DARPA Wants Your Insect-Scale Robots for a Micro-Olympics, 18 Jul 2018, Available Online: https://spectrum.iee.org/automaton/robotics/robotics-hardware/darpa-wants-your-insect-scalerobots-for-a-micro-olympics?utm_source=roboticsnews\&utm_campaign=roboticsnews-07-3118\&utm_medium=email

[9] Richard Moss, 3D-printed microscopic fish could be forerunners to smart "microbots", New Atlas,August 26th, 2015, Available Online: https://newatlas.com/3d-printed-microscopic-fish-smart-microbots/39106/

[10] Field Systems,

Fanuc

2018

Available

Online: https://www.fanuc.co.jp/en/product/catalog/pdf/field/FIELDsystem(E)-01.pdf

[11] SAM FRANCIS, Kuka to build global deep learning AI network for industrial robots, Robotics and Automation News, MARCH 19, 2016, Available Online: https://roboticsandautomationnews.com/2016/03/19/kuka-to-build-global-iot-network-and-deeplearning-ai-system-for-industrial-robots/3570/

[12] FORBES INSIGHTS With Intel AI, How AI Builds A Better Manufacturing Process, Forbes, Jul 17, 2018, Available Online: https://www.forbes.com/sites/insights-intelai/2018/07/17/how-ai-builds-abetter-manufacturing-process/\#2ab59f1b1e84 
International Journal of Control and Automation Vol. 12, No. 1 (2019) 